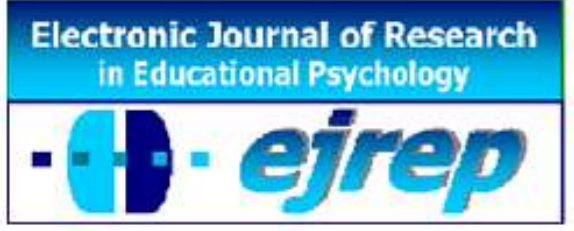

\title{
Perfiles de Resiliencia y Estrategias de Afrontamiento en la Universidad: Variables Contextuales y Demográficas
}

\section{M $^{\text {a }}$ Carmen González Torres ${ }^{1}$, Raquel Artuch Garde ${ }^{1}$}

${ }^{1}$ Facultad de Educación y Psicología. Universidad de Navarra.

\section{España}

Correspondencia: María Carmen González-Torres. Departamento de Teoría y Métodos en Educación y Psicología. Facultad de Educación y Psicología. Universidad de Navarra. e-mail: mgonzalez@unav.es (C) Education \& Psychology I+D+i and Ilustre Colegio Oficial de Psicología de Andalucía Oriental 


\section{Resumen}

Introducción. En las dos últimas décadas y en relación con la educación de las llamadas competencias del S.XXI se está prestando una atención creciente al papel desempeñado por los factores no cognitivos en el aprendizaje, el éxito académico, social y profesional. En este marco hay dos variables muy interrelacionadas, resiliencia y afrontamiento que son determinantes de la conducta frente al estrés en contextos académicos y sólo recientemente han empezando a ser objeto de atención en el ámbito universitario.

Método. Un total de 117 alumnos universitarios participaron en este estudio en el que se aplicó la escala de afrontamiento EEC y la escala de resiliencia CD-RISC. Se realizaron análisis descriptivos, de asociación y MANOVAS para comprobar el perfil resiliente y de estrategias de afrontamiento ante el estrés de los alumnos, observar el tipo de relación entre ambos constructos y analizar el posible efecto de las variables sexo y tipo de universidad sobre su perfil resiliente y de estrategias de afrontamiento.

Resultados. Se destaca una puntuación media-alta en resiliencia y un uso general de estrategias de afrontamiento centradas en el problema. Se resalta la relación positiva y significativa entre las variables analizadas y se confirman efectos del sexo y tipo de universidad sobre factores como tenacidad, espiritualidad, autoinstrucciones, acción dirigida a las causas, reevaluación positiva y firmeza y apoyo religioso entre otros.

Discusión y conclusiones. Los resultados proporcionan evidencia acerca de las conexiones entre resiliencia y las estrategias de afrontamiento en este ámbito y ofrecen información de interés sobre cómo algunos indicadores de ambas variables son sensibles a la influencia del género y del contexto (tipo de universidad). Se destaca la necesidad de seguir profundizando en estas cuestiones y se apunta su interés para los servicios de admisión y orientación universitarios.

Palabras Clave: resiliencia, estrategias de afrontamiento, universidad, género. 


\title{
Profiles of resilience and coping strategies at university: contextual and demographic variables
}

\begin{abstract}
Introduction. In the past two decades and increase attention is been paid in the importance of non cognitive factors in learning, and the academic social and professional success. There are two different variable interrelated: resilience and coping strategies which influence the behavior against the stress in the academic context, and only lately university professionals have paid attention on them.

Method. 117 university students took part in that research, where EEC coping and CD-RISC resilience scales where used. Descriptive and association analysis as well as MANOVAs where realized to make sure the resilient profile, and the strategies that need to be used in order to help students with their stress, observe the relationship between both constructs and analyze the possible effect that the gender or the kind of university may have in the students resilient profile, and in the coping strategies.
\end{abstract}

Results. High average score in resilience and the general use of coping strategies, which are focused in the problem, are underlined in the research. Not only the connection between the variables is important but also it is proved the effects that the gender and the university have in the perseverance, steadiness, spirituality, positive re-evaluation and religious support.

Discussion and Conclusions. The research saws the evidence that there is a connection between resilience and coping strategies and offer important information about how some indicators can be influence by the gender and the context, such as the type of university. It is believed the necessity to underline that more research should be done. The results are important, especially for the admission and orientation departments.

Keywords: noncognitive skills, resilience, coping strategies, college, genre 


\section{Introducción}

En los debates actuales desde instancias políticas, económicas, sociales, educativas y de investigación sobre las competencias claves para el S.XXI - conocidas como 21st Century skills (Suto, 2013; Costa y Kallick, 2014)-, son constantes las referencias a la necesidad de potenciar en los estudiantes un conjunto de variables de carácter disposicional que se recogen bajo la rúbrica de "factores no cognitivos". También son conocidos con otras denominaciones como life skills, soft skills, personal skills, fortalezas de carácter (character skills/strensgths), disposiciones, hábitos de la mente, aprendizaje socioemocional, entre otros (Conley, 2013; Dweck, Walton, y Cohen, 2011; Egalité; Mills y Greene, 2014; Gutman y Schoon, 2013; Heckman y Rubinstein, 2001; Rosen, Glennie, Dalton, Lennon y Bozick, 2010).

\section{Importancia de los factores motivacionales-afectivos en el aprendizaje}

Con el término factores no cognitivos (softs skills) los autores se refieren a un conjunto de actitudes, conductas, estrategias, valores, creencias, rasgos de personalidad que favorecen el éxito en la escuela, la universidad y en el mundo del trabajo y que abarcan una miscelánea de aspectos diversos no fácilmente diferenciables (Heckman y Rubinstein, 2001) tales como motivación, perseverancia, optimismo, autocontrol, tenacidad, diligencia, demora de la gratificación, autodisciplina, resiliencia, fuerza de voluntad, habilidades sociales y un largo etc. Aunque se ha agigantado su interés en los últimos años, este concepto no es nuevo, fue introducido por los sociólogos Bowles y Gintis en 1976 (Gutman y Schoon, 2013) y se usa en contraste con las llamadas hard skills referidas a las habilidades cognitivas que se suelen medir convencionalmente en los test cognitivos o pruebas de rendimiento académico.

Son abundantes los estudios que desde diferentes disciplinas han mostrado su asociación con los logros académicos, el éxito interpersonal y en el mundo del trabajo (DeRidder, Lensvelt-Mulders, Finkenauer, Stok y Baumeister, 2012; Duckworth y Seligman, 2005; Durlak, Weissberg, Dymnicki, Taylor, Schellinger, 2011; Heckman, Stixrud, y Urzua, 2006;West, Kraft, Finn, Duckworth, Gabrieli y Gabrieli, 2014).

Para muchos son tan importantes o incluso más que las habilidades cognitivas o el CI (Duckworth y Seligman, 2005; Heckman y Rubinstein, 2001), aunque este tema no deja de ser polémico al igual que el término "factores no cognitivos". No parece muy afortunado puesto que crea una falsa dicotomía entre las habilidades cognitivas y estas variables de carácter psicosocial (soft skills) cuando, como apuntan Borghans, Duckwort, Heckman y ter 
Weel (2008), pocos aspectos de la conducta humana están excluidos de cognición. Se aboga entonces por repensar esta noción de factores no cognitivos (Conley, 2013; Gutman y Schoon, 2013) y también por avanzar más en el desarrolla de medidas rigurosas de estas variables para que sean tan potentes como las medidas de las habilidades cognitivas de forma que su uso se extienda para conocer mejor lo que el alumno aprende y cómo lo aprende. De acuerdo con Conley, una mejor información de este "dominio no cognitivo" ayudará a los alumnos a gestionar su propio aprendizaje y ser más dueños del mismo.

Se han desarrollado muchas y muy diversas listas de factores no cognitivos (Costa y Kallick, 2014). Muchos de los cuales tienen alto grado de solapamiento. En este trabajo nos vamos a ocupar de la resiliencia y de las estrategias de afrontamiento en estudiantes universitarios. Antes de entrar más de lleno en esta cuestión queremos destacar como botón de muestra algunos trabajos y líneas de investigación que están contribuyendo a acentuar la importancia de invertir en el contexto educativo en el desarrollo de estas y otras variables no cognitivas muy relacionadas.

Uno de los autores que más ha popularizado el término no cognitivo ha sido el premio nobel de economía James Heckman (Heckman y Rubinstein, 2001) para quien más allá del conocimiento y las habilidades académicas y técnicas, factores como la motivación, la gestión del tiempo y la auto-regulación son críticos para los logros en la vida incluido el éxito en el mercado laboral.

Duckworth y sus colaboradores llevan varios años dedicados al estudio del autocontrol (sinónimo de autodisciplina /fuerza de voluntad) y al constructo denominado grit (perseverancia/coraje) (Duckwork y Carlson, 2013; Duckworth y Seligman, 2005; Duckworth, Peterson, Matthews, Kelly, (2007). Duckworth y Carlson (2013), en su revisión sobre autocontrol y éxito escolar, concluyen que la evidencia empírica muestra que el aprendizaje, la aplicación de las habilidades cognitivas y del conocimiento, la permanencia en la escuela y graduarse en la secundaria y la universidad dependen en gran medida de ese control voluntario de los impulsos atencionales, emocionales y conductuales al servicio de metas y estándares personalmente valorados. Por su parte, Dweck (2006) muy conocida por sus estudios sobre el papel de la concepción de la inteligencia fija o maleable en la motivación y en el aprendizaje, ha desarrollado el amplio concepto de "tenacidad académica" (academic tenacity) (Dweck, Walton y Cohen (2011) y también se interesa por analizar el 
impacto de la visión incremental/estable de la inteligencia en la resiliencia de los estudiantes frente a los desafíos académicos y sociales (Yeager y Dweck, 2012).

Farrington y Farrington, Roderick, Allensworth, Nagaoka, Keyes, Johnson y Beechum, 2012 han realizado una amplia revisión auspiciada por The University of Chicago Consortium on Chicago School Research (CCSR) en la que, a partir del examen de cientos de estudios de factores de carácter no cognitivo ligados con el éxito académico, establecen una categorización de los mismos señalando su importancia para el éxito en la educación postsecundaria y universidad. Entre ellos destacamos el que denominan academic perseverance que tiene que ver con la resiliencia y recoge un amplio cuerpo de conceptos psicológicos como los propuestos por Duckwortk y Dweck que hemos mencionado. En su revisión definen la perseverancia académica como "la habilidad del estudiantes para permanecer centrado e implicado en el trabajo a pesar de las distracciones, contratiempos $\mathrm{u}$ obstáculos”. Recalcan que en el contexto universitario ésta es particularmente importante.

Gutman, y Schoon, (2013) han presentado una revisión en el Institute of Education de la Universidad de Londres sobre el impacto de las habilidades no cognitivas en los logros de niños y jóvenes. Uno de sus objetivos ha sido identificar competencias claves que sean flexibles y maleables y proponen 8 factores. Uno de ellos lo denominan resiliencia y afrontamiento. En España De la Fuente y su equipo (De la Fuente, 2014a y 2014b; De la Fuente, Solinas, Fadda y Zapata, in review) llevan varios años trabajando en un proyecto de investigación sobre estrategias motivacionales -afectivas que favorecen la autorregulación personal y el afrontamiento en el proceso de enseñanza-aprendizaje de la universidad de la que se ha derivado una plataforma online de autoevaluación para los alumnos y una guía para aprender, estudiar y rendir bajo estrés.

Guy Claxton es otro de los autores que desde Inglaterra más se ha hecho eco a través de sus libros, artículos, conferencia y con su organización (www.buildinglearningpower.co.uk) del rol de las virtudes, disposiciones, hábitos, cualidades de la mente en el aprendizaje y el desarrollo pleno de los jóvenes. En su libro The Learning Powered School, Claxton, Chambers, Powel y Lucas (2011) proponen una taxonomía de 17 disposiciones a modo de músculos del aprendizaje que las clasifican en cuatro categorías en su modelo 4'R of Learning Power (Resiliencia, Ingeniosidad (resourcefulness), Reflexividad (reflectiveness) y reciprocidad). También a caballo entre la investigación y divulgación científica el libro de Tough (2013) How children succeed: grit, Curiosity and the Hidden 
Power of Character ha tenido una notable repercusión en ámbitos científicos, sociales e incluso en la política educativa estadounidense (Costa y Kallick, 2014).

Sin duda a este renacimiento del interés por los factores no cognitivos han contribuido enormemente líneas importantes de investigación que tienen una larga tradición como la de los investigadores del Proyecto Zero de la Harvard Graduate School of Education sobre las disposiciones del pensamiento o el carácter intelectual (Perkins y Tishman, 2001; Ritchhart, 2001), las aportaciones de Costa y Kallich (2000 y 2014) sobre los 16 hábitos de la mente, la investigación en el campo del aprendizaje autorregulado sobre la dimensión afectivamotivacional-volitiva del mismo (will) y los trabajos sobre estrategias de control motivacional y volitivo (Wolters, 2003; González-Torres, 2012). Por otra parte, la larga tradición de estudio sobre resiliencia y su visión optimista sobre las posibilidades de todos los individuos para hacer frente con éxito a la adversidad se ha unido con fuerza a las investigaciones, objetivos y metas de la Psicología Positiva (Chung, 2011; Masten, 2004; Vargas y González-Torres; Yates, 2009). Chung (2011) señala que las virtudes y fortalezas de carácter propuestas por la psicología positiva pueden ser la base de la resiliencia.

\section{La resiliencia y su interés en el contexto educativo general y universitario en particular}

La resiliencia es un constructo complejo. No es una realidad nueva pero su investigación científica en el ámbito de la psicología empezó en los años 50 y los hallazgos iniciales se presentaron en los años 80 del s. XX (Smith Osborne, 2007).

Las diferentes definiciones de resiliencia destacan que es la habilidad o más bien los procesos que capacitan a los individuos para enfrentarse con éxito a los eventos estresantes o adversos que aparecen en la vida de modo que se crece en este proceso, se gana en competencia (enriquecimiento de las cualidades resilientes o factores de protección) y adaptación (Artuch, 2013; Luthar, Cicchetti y Becker, 2000; Luthar, Sawyer y Brown, 2006). Para que se pueda hablar entonces de resiliencia, tal como nos recuerdan Masten y Coatsworth (1998) es necesario: 1) la presencia de una amenaza, riesgo o adversidad en la vida del individuo y que 2) la calidad de la adaptación frente al riesgo o adversidad sea evaluada como buena.

Su valor educativo es enorme ya que invita al optimismo pedagógico pues, como la investigación ha mostrado, no es algo particular de unas pocas "personas invencibles o invulnerables" como se pensó en la primera etapa de investigación de este constructo (Werner 
y Smith, 1982) sino que más bien todos somos robust-yet-fragile, RYF (Zolli y Healy, 2012). Como señaló Masten (2001), la resiliencia es "la magia de lo ordinario" (ordinary magic). Su estudio en el ámbito educativo y sobre todo en relación a niños y adolescentes se ha incrementado en los últimos años (Artuch, 2013; Doll, Zucker y Brehm, 2014; Goldstein y Brooks, 2013; González-Torres, 2011; Jordan, 2010, Martin y Marsh, 2006; Prince-Embury y Saklofske, 2013; Waxman, Gray y Padrón, 2003).

Puesto que la resiliencia no es una cualidad especial de algunos individuos sino que todos tenemos el potencial de ser resilientes se ha dedicado mucha investigación a conocer los factores de protección interna y externa que la favorecen. Una lista de factores de protección muy conocida en el contexto educativo es la propuesta por Benard (1991). Esta autora en sus estudios -y corroborado por otros muchos (Artuch, 2013)- encontró que los niños y jóvenes en riesgo pero resilientes mostraron las siguientes características internas: competencia social (flexibilidad, empatía, cuidado, habilidades de comunicación, sentido del humor); capacidades de resolución de problemas (planificación, búsqueda de ayuda, pensamiento crítico y creativo), autonomía (sentido de identidad, autoeficacia, autoconsciencia, sentido de dominio y alejamiento de los mensajes y condiciones negativas), sentido de propósito y la creencia en un futuro positivo (aspiraciones educativas, optimismo, fe y conexión espiritual). Como factores de protección externa señaló: cuidado e implicación afectiva de adultos, altas expectativas y participación y contribución a la comunidad.

Werner (2007) sugiere que los factores de protección de la resiliencia parecen ser universales a través de las culturas, razas y estratos socioeconómicos; pero, sin embargo, como es un proceso más que un rasgo, la efectividad de los mismos y el comportamiento y la respuesta resiliente son específicos. Por ejemplo, la respuesta resiliente dada por los adolescentes puede variar, como señalan Fergus y Zimmerman (2005), dependiendo del tipo de factores de riesgo a los que se enfrente, la interpretación que hagan de ellos, el lugar de residencia (ámbito urbano/rural), estar en una etapa de la adolescencia u otra, ser chico o chica.

Patterson (2002) reconoce que con la proliferación de investigaciones sobre resiliencia y sus aplicaciones en la práctica se ha producido una cierta confusión respecto a su definición y a la decisión sobre quién es resiliente, lo cual depende de la visión de la naturaleza y extensión de lo que se considera situación adversa o de riesgo. Este autor distingue dos perspectivas sobre la resiliencia. Desde una se vería que la exposición a situaciones de alto 
riesgo (riesgos significativos) sería un prerrequisito para poder ser considerado resiliente si la adaptación es positiva; desde otra, se podría aplicar a todos los que desarrollan fortalezas y funcionan competentemente en muchas circunstancias vitales puesto que la vida en general ya es lo suficientemente desafiante como para crear exposición al riesgo (perspectiva de life asrisk).

Mientras que inicialmente en el campo educativo las investigaciones se centraron en el estudio de estudiantes de alto riesgo (p.e. riesgo de fracaso y exclusión social; niños con discapacidades o problemas de aprendizaje severo; niños en situaciones familiares o sociales muy adversas); sin embargo, aunque no sin controversia (Martin y Marsh, 2008), ha ido calando la segunda visión señalada por Patterson. Desde esta perspectiva un evento traumático no es un antecedente necesario para la conducta resiliente (Davino, 2013). Así se podría hablar de resiliencia cuando se afrontan los desafíos propios de los distintos momentos evolutivos (p.e. paso de la niñez, a pubertad, juventud) y los que se pueden encontrar en el aprendizaje escolar o universitario.

Se ha desarrollado, como hemos señalado, una amplia investigación sobre la resiliencia en niños y adolescentes pero los estudios en población universitaria son todavía escasos (Davino, 2013; Hassim, Strydom y Stridom, 2013; Johnson, 2011; McCann y Hicks, 2011; McLafferty, Mallet y McCauley, 2012; Munro y Pooley, 2009; Prynyapol, 2003; Terzi, 2013) y, sin embargo, la vida universitaria es un contexto importante de estrés donde los estudiantes se enfrentan a muchos cambios y desafíos muy diferentes del de otros grupos (Steinhart y Dolbier, 2008) que pueden crear vulnerabilidad y afectar a su adaptación y éxito académico (De la Fuente 2014a y 2014b). Así, además de los retos vinculados con la transición de la adolescencia a la primera adultez (lucha por mayor independencia, dar forma a su identidad personal y profesional, avanzar en búsqueda de significado para sus vidas) se tienen que adaptar a nuevas estructuras y a una cultura con unas demandas académicas y sociales muy diferentes a las que estaban acostumbrados. Tienen que hacer frente a un conjunto de estresores de muy diverso tipo: intrapersonales (cambios en los hábitos de sueño y comida, nuevas responsabilidades), interpersonales (actividades sociales, relaciones románticas y sus conflictos), académicos (exámenes, sobrecarga de clase, estudio, prácticas), ambientales (instalaciones de la universidad, problemas con las TIC.) (Davino, 2013; Dziegielwski, Turnage y Roest-Marti, 2004; Ross, Niebling y Heckert , 1999; Steinhart y Dolbier, 2008). 


\section{Resiliencia y afrontamiento en el ámbito universitario}

Se ha propuesto que el ajuste y éxito académico en la universidad requiere altos niveles de resiliencia (Munro y Pooley, 2009) y por ello, se está empezando a prestar más atención al estudio de los factores que protegen y contribuyen a fomentarla a través de toda la vida universitaria (De la Fuente, Cardelle-Elawar, Matinez-Vicente, Zapata y Peralta, 2013).

Se han señalado muchos factores de protección interna de la resiliencia y entre ellos cabe destacar su estrecha conexión con las estrategias de afrontamiento (coping) (Glennie, 2010; Guttman y Shoon, 2013). Pocos estudios han investigado las relaciones entre estilos de afrontamiento y resiliencia en población universitaria (De la Fuente, Cardelle-Elawar Matinez-Vicente, Zapata y Peralta, 2013; Orozco, 2007; Li, in press, Terzi, 2013).

Por otra parte, todavía se conoce poco acerca de cómo "el clima del campus" (Orozco; 2007; Terzi, 2013) y las diferencias de género influidas por los procesos culturales y de socialización conducen a diferencias en los indicadores de resiliencia y afrontamiento, aunque se ha postulado en la literatura que la resiliencia es una característica multidimensional y específica que varía en función de las características de la población, el tipo de contenido, la edad, el género, el contexto, el momento evolutivo y otras variables (Connor, Davidson y Lee, 2003; De la Fuente y cols. 2013; Fergus y Zimerman, 2005; Luthar, Ciccetti y Becker, 2000; Werner, 2007; Zolkoski y Bullock, 2012). Se plantea que hombres y mujeres comparten muchas influencias ambientales pero también que son socializados de acuerdo a diferentes patrones que pueden afectar a la forma en que se sitúan frente a las experiencias estresantes y al uso que hacen de los recursos de afrontamiento (Johnson, 2011).

Coping y resiliencia se han utilizado de forma intercambiable aunque son constructos diferentes - la resiliencia se refiere a los resultados positivos en respuesta a una situación estresante mientras que coping alude a las estrategias empleadas para manejar los efectos de la situación de estrés (Glennie, 2010; Guttman y Shoon, 2013; Orozco, 2007). De acuerdo con Lazarus y Folkman (1984), las respuestas de afrontamiento son los esfuerzos cognitivos y conductuales que los individuos emplean para dominar, tolerar o reducir los efectos de los sucesos vitales estresantes. En la literatura se distinguen dos tipos de afrontamiento: centrado en el problema y centrado en la emoción, aunque se cuestiona esta delimitación (De la Fuente y cols., 2013). En el afrontamiento centrado en el problema (o activo), que puede ser cognitivo o conductual, los intentos de los individuos se dirigen a manejar o alterar las fuentes 
de estrés e incluye establecer un plan de acción, buscar nuevos recursos, buscar ayuda, reinterpretar el significado de un fracaso, entre otros. En el afrontamiento centrado en la emoción el individuo emplea estrategias para manejar o reducir su malestar (distress) emocional asociado con la situación estresante. Las personas tienden a utilizar más las estrategias centradas en el problema cuando la situación se percibe alterable y más las centradas en la emoción cuando se percibe inalterable (Peralta, 2013).

En la escasa literatura relativa a resiliencia y afrontamiento en el ámbito universitario los estudios indican que la resiliencia está significativamente relacionada con el uso de estrategias de afrontamiento activo (centrado en el problema), que el afrontamiento centrado en la emoción está más asociado con altos niveles de estrés y que el estilo de evitación es más característico de los estudiantes no resilientes (De la Fuente y cols. 2013; Li, 2008, Li, en prensa; Orozco, 2007; Sagone y De Caroli, 2014; Steinhart y Dolbier, 2008; Terzi, 2013). Sin embargo, también se ha señalado que no hay ningún estilo de afrontamiento que sea funcional y efectivo en todas las situaciones (Orozco, 2007). Por su parte, Campbell-Sills, Cohan, y Stein (2006) encontraron que tanto las estrategias centradas en la emoción como en el problema fueron predictores significativos de resiliencia.

Las diferencias de género en resiliencia y coping se han estudiado más en la secundaria obligatoria y menos en primaria y universidad (De la Fuente y cols., 2013). Respecto a las diferencias de género en afrontamiento los resultados son inconsistentes ( Zapata, 2013). Algunos no encuentran diferencias (McLafferty, Mallet y McCauley, 2012), otros estudios indican que no hay claras diferencias de género generales aunque las chicas parecen hacer más uso de estrategias de afrontamiento centradas en el problema (De la Fuente y cols., 2013; Zapata, 2013). Otros estudios encuentran diferencias indicando que las chicas tienden a usar más la búsqueda de apoyo social y las estrategias centradas en la emoción como comunicar los sentimientos (Sagone y De Caroli, 2014).

Por otra parte, hay estudios que indican que las mujeres muestran más altos niveles de estrés y más bajos niveles de resiliencia que los hombres (Li, 2008; Li, en prensa; Johnson, 2010; McLaffertty y cols, 2012). Sin embargo, otros estudios no encuentran diferencias de género generales (De la fuente y cols., 2013; Leary y DeRosier, 2012; McLafferty, Mallet y McCauley, 2012) Como vemos los resultados son también inconsistentes y se precisa más investigación. 


\section{Objetivos e hipótesis}

El propósito de este trabajo es estudiar la resiliencia en el contexto de la educación superior y su relación con las estrategias de afrontamiento. Se pretende aportar datos que confirmen la relación entre la capacidad resiliente de las personas y sus estrategias de afrontamiento ante situaciones vivenciales de estrés, en este caso, en el contexto universitario. Por ello queremos observar: a) el perfil de resiliencia y de estrategias de afrontamiento general de la muestra; b) relación entre la resiliencia y sus factores y los dos tipos de estrategias de afrontamiento; c) observar el efecto de variables contextuales como el tipo de Universidad (LAICA, pública-RELIGIOSA, privada) (VI) o variables personales como el Género (Masculino-Femenino) (VI) en la resiliencia (VD) y en las estrategias de afrontamiento ante el estrés (VD). De manera consecuente, las hipótesis del estudio fueron:

H1: La puntuación en resiliencia y de estrategias de afrontamiento de los alumnos será media, según estudios realizados con poblaciones similares. H2: Las estrategias de afrontamiento centradas en el problema tendrán una relación positiva y significativa con la escala de resiliencia, ya que como señalan las investigaciones las estrategias de coping son parte del elenco de factores protectores resilientes; H3: Existirá un efecto del género y el tipo de universidad sobre la resiliencia y las estrategias de afrontamiento adoptadas por los alumnos en diferentes situaciones de estrés académico.

\section{Método}

\section{Participantes}

De un total de 176 alumnos fueron 117 los estudiantes que conformaron la muestra final de nuestro estudio porque completaron correctamente todas las pruebas. Procedían de una universidad laica $(66.7 \% ; n=78)$ y una universidad religiosa $(33.3 \% ; n=39)$ de los primeros curos de los Grados de Educación y Psicología. La media de edad de los participantes fue de 20.55 (DT=4.52) con un rango de 18 a 60 años, siendo el rango de 19 a 20 años el de mayor porcentaje del alumnado (56.4\%). Entre los participantes encontramos a mujeres $(62.4 \% ; n=73)$ y hombres $(33.3 \% ; n=39)$ que cursan asignaturas psicología de la educación en las respectivas universidades. 


\section{Instrumentos}

La resiliencia se evaluó con la Escala CD-RISC (Connor y Davidson, 2003) en en su versión traducida al castellano (Bobes, Bascarán, García-Portilla, Bousoño, Saiz, Wallace y Hidalgo, 2001 y 2008). Esta escala, una de las más utilizadas en los estudios sobre resiliencia (Windle, Bennett y Noyes, 2011), consta de 25 ítems estructurados en una escala sumativa tipo Likert compuesta por 5 factores: F1: Tenacidad y competencia personal, F2: confianza en la intuición y tolerancia al estrés, F3: aceptación positiva del cambio, F4: percepción de control y F5: espiritualidad. El cuestionario presenta una fiabilidad Alfa de Cronbach de .89.

Las estrategias de afrontamiento fueron evaluadas con la Escala de Estrategias de Coping, EEC (Chorot y Sandín, 1993) en su versión traducida y adaptada al castellano (De la Fuente, 1994, 2011). Esta escala está basada en el modelo de Lazarus y Folkman (1984) y adaptada para medir las estrategias de afrontamiento ante situaciones de estrés para estudiantes universitarios. Está compuesta por un total de 90 ítems agrupados en 13 factores que se estructuran en dos dimensiones: estrategias de afrontamiento centrado en la emoción que contiene 7 factores -F1. Distracción fantástica; F4. Apoyo religioso; F6. Ayuda para la acción; F7. Reducción de la ansiedad y evitación; F8. Prepararse para lo peor; F9. Descarga emocional y aislamiento; F11. Aceptación resignada- y estrategias de afrontamiento centradas en el problema formada por 6 factores -F2. La búsqueda de ayuda; F3. Acciones dirigidas a las causas; F5. Autoinstrucciones; F10. Reevaluación y firmeza positiva; F12. Comunicación de los sentimientos y apoyo social; F13. Búsqueda de refuerzo alternativo-. La escala completa obtuvo una fiabilidad de 0,93 (Alfa de Cronbach).

\section{Procedimiento}

Los alumnos, cuya participación fue voluntaria y a quienes se les garantizó la confidencialidad de los datos, cumplimentaron las pruebas a través de una plataforma online denominada e-Afrontamiento del Estrés Académico (de la Fuente, 2014) y en papel. La recogida de datos se realizó durante el curso académico 2013-2014.

Este estudio forma parte del Proyecto I+D Estrategias motivacionales-afectivas de autorregulación personal y de afrontamiento del estrés en el proceso de enseñanzaaprendizaje universitario EDU2011-24805, por lo que los alumnos cumplimentaron más pruebas además de las descritas en este artículo. 


\section{Análisis de datos}

Se utilizó un diseño ex post-facto. Se realizaron análisis de Asociación con correlaciones bivariadas de Pearson, análisis de varianza ANOVAS y multivariados MANOVAs, con Scheffé post hoc, y tamaño del efecto. Los análisis que se llevaron a cabo para cumplir con los objetivos propuestos y probar las hipótesis planteadas se realizaron utilizando el paquete estadístico SPSS versión 21.00 para Windows.

\section{Resultados}

Perfil resiliente y estrategias de afrontamiento en estudiantes universitarios

La media de la puntuación total en la escala de resiliencia fue de M=3.67 (DT=0.43) sobre 5 mientras que la media de las dimensiones afrontamiento centrado en la emoción y afrontamiento centrado en el problema fue de $\mathrm{M}=2.28$ ( $\mathrm{DT}=0.33$ ) y $\mathrm{M}=2.93$ ( $\mathrm{DT}=0.37)$ sobre 4 respectivamente.

En la escala de resiliencia se observan mayores puntuaciones en los factores control y cambio para los cuales encontramos puntuaciones significativamente mayores que el factor espiritual $[t(116)=32.137, p<0.001]$ (ver Gráfico 1$)$.

Para la muestra total de alumnos los factores que obtuvieron mayor puntuación en la escala de afrontamiento (ver Gráfico 2) fueron reevaluación positiva y firmeza y autoinstrucciones de la escala de afrontamiento EEC. Los dos factores fueron significativamente mayores que el factor descarga emocional $[t(116)=53.120, p<0.001]$ y apoyo religioso $[t(116)=, p<0.001]$ que obtuvieron la menor puntuación. Se vislumbra un uso de estrategias de afrontamiento orientadas al problema frente a aquellas centradas en la emoción. 


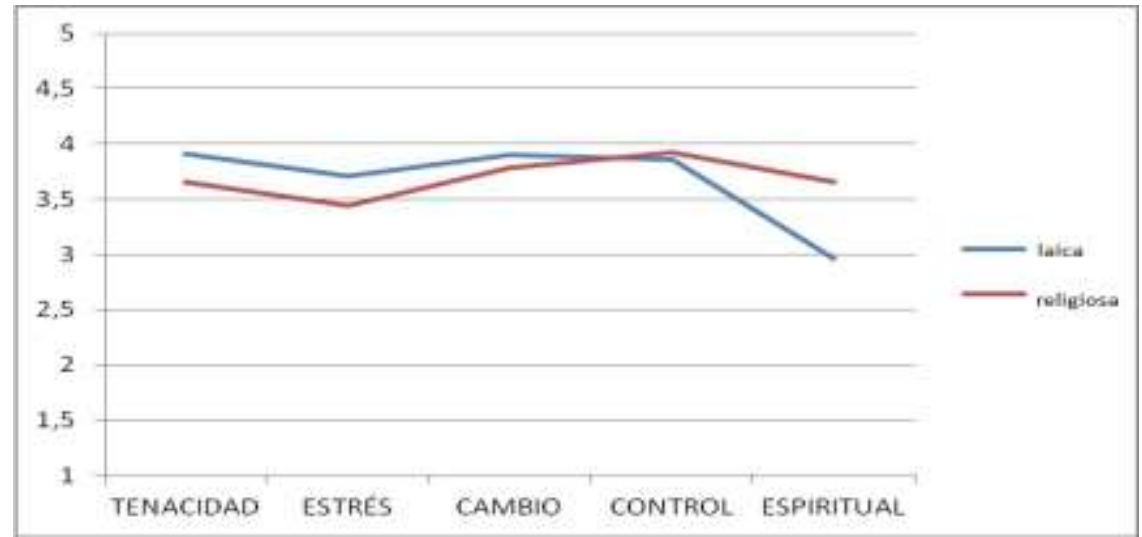

Gráfico 1. Perfil de resiliencia de los alumnos de la universidad laica y universidad religiosa

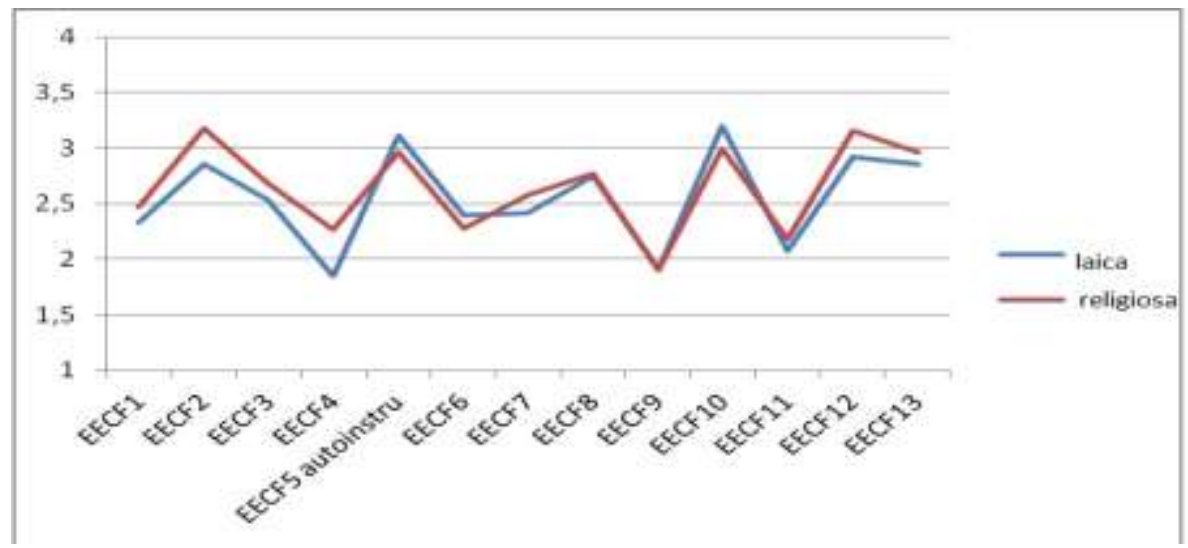

Gráfico 2. Perfil de estrategias de afrontamiento de los alumnos de la universidad laica y universidad religiosa

Relación entre la resiliencia y las estrategias de afrontamiento

Se observa una relación positiva y significativa al nivel de $p<.001$ entre las estrategias de afrontamiento centradas en el problema y la resiliencia, excepto en el factor espiritual de la escala de resiliencia en el que se observa mayor relación con el afrontamiento centrado en la emoción.

La relación entre los factores apoyo religioso y espiritual de ambas escalas fue la más alta $(r=.633, p<.001)$, seguida de la existente entre los factores reevaluación positiva y firmeza y tenacidad $(r=.535, p<.001)$.

Todos los factores de resiliencia (tenacidad, estrés, cambio y control) a excepción del factor espiritual, correlacionan positiva y significativamente con los factores 
autoinstrucciones y reevaluación positiva y firmeza que representan un afrontamiento centrado en el problema (ver tabla 1).

Tabla 1. Relación entre factores de resiliencia y factores de afrontamiento

\begin{tabular}{lcccc}
\hline & TENACIDAD & ESTRÉS & CAMBIO & CONTROL \\
\hline $\begin{array}{l}\text { Autoinstrucciones } \\
\begin{array}{l}\text { Reevaluación } \\
\text { positiva y firmeza }\end{array}\end{array}$ & $\mathbf{4 5 6}^{* *}$ &, $304^{* *}$ &, $320^{* * *}$ &, $335^{* * *}$ \\
\hline
\end{tabular}

Interacción entre la resiliencia y el sexo x la universidad

Los resultados MANOVA efectuados entre la puntuación total de resiliencia y la interacción sexo x universidad ponen de manifiesto para esta muestra la inexistencia de efecto de ambas variables independientes juntas sobre la puntuación global de resiliencia (VD). Tampoco se observa un efecto del sexo ni del tipo de universidad en la puntuación global de resiliencia.

No se detecta efecto del sexo sobre ninguno de los factores de resiliencia. Sin embargo, sí se observa un efecto del tipo de universidad (laica-religiosa) sobre algunos factores relacionados con la resiliencia como tenacidad $\left[F(1,113)=4916, p<0.05\right.$, eta $\left.{ }^{2}=.042\right]$ y espiritualidad $\left[F(1,113)=10059, p<0.005\right.$, eta $\left.{ }^{2}=.082\right]$ (Ver tabla 2).

Tabla 2. Estadísticos descriptivos de factores de la escala de resiliencia

\begin{tabular}{|c|c|c|c|c|c|c|c|}
\hline \multicolumn{8}{|l|}{ FACTORES } \\
\hline \multirow[t]{2}{*}{ F. Tenacidad } & \multicolumn{7}{|c|}{ F. Espiritual } \\
\hline & Laica & Religiosa & Total & & Laica & Religiosa & Total \\
\hline Hombre & $3.95(.66)$ & $3.45(.73)$ & $3.90(.68)$ & Hombre & $2.79(1.19)$ & $4.10(.82)$ & $2.92(1.22)$ \\
\hline Mujer & $3.86(.50)$ & $3.68(.49)$ & $3.77(.50)$ & Mujer & $3.17(.92)$ & $3.58(.87)$ & $3.38(.91)$ \\
\hline Total & $3.91(.59)$ & $3.65(.52)$ & & Total & $2.96(1.09)$ & $3.65(.87)$ & \\
\hline
\end{tabular}

Interacción entre estrategias de afrontamiento y sexo $x$ universidad

El MANOVA efectuado entre la universidad y el sexo y las dos dimensiones de estrategias de afrontamiento pone de manifiesto un efecto de la interacción universidad $x$ sexo sobre la dimensión estrategias de afrontamiento centradas en el problema $[F(1,113)$ $\left.=6181, p=0.014, e t a^{2}=.052\right]$. 
Se observa también efecto de la variable sexo sobre la dimensión estrategias de afrontamiento centradas en el problema $\left[F(1,113)=22785, p<0.001\right.$, eta $\left.{ }^{2}=.168\right]$ en las que destacan las chicas. En cuanto al efecto del tipo de universidad en la que se cursen los estudios (laica/religiosa) se observa un efecto de ésta sobre la dimensión estrategias centradas en el problema $\left[F(1,113)=4110, \mathrm{p}<0.05\right.$, eta $\left.^{2}=.035\right] .($ Ver tabla 3$)$

Tabla 3. Estadísticos descriptivos para el tipo de universidad y sexo en la dimensión afrontamiento centrado en el problema

\begin{tabular}{rrrr}
\hline Dimensión: Afrontamiento centrado en el problema & \\
\hline & Laica & Religiosa & Total \\
Hombre & $2.82(.40)$ & $2.40(.43)$ & $2.77(.41)$ \\
Mujer & $3.03(.29)$ & $3.07(.30)$ & $3.05(.29)$ \\
Total & $2.91(.37)$ & $2.98(.39)$ & \\
\hline
\end{tabular}

Se detecta un efecto de la interacción universidad x sexo sobre los factores de acción dirigida a las causas $\left[F(1,113)=5133, p<0.05\right.$, eta $\left.{ }^{2}=.043\right]$ y búsqueda de refuerzo alternativo $\left[F(1,113)=6542, p<0.05\right.$, eta $\left.^{2}=.055\right]$. También un efecto del sexo (varón, mujer) sobre los factores búsqueda de ayuda $\left[F(1,113)=15029, p<0.001\right.$, eta $\left.{ }^{2}=.117\right]$, acción dirigida a las causas $\left[F(1,113)=20567, p<0.001\right.$, eta $\left.{ }^{2}=.154\right]$, autoinstrucciones $[\mathrm{F}(1,113)$ $=5177, p<0.05$, eta $\left.{ }^{2}=.044\right]$, ayuda para la acción, $\left[\mathrm{F}(1,113)=6225, p<0.05\right.$, eta $\left.{ }^{2}=.052\right]$, preparación para lo peor $\left[F(1,113)=5158, p<0.05\right.$, eta $\left.{ }^{2}=.044\right]$, comunicación de sentimientos $y$ apoyo social $\left[F(1,113)=18708, p<0.001\right.$, eta $\left.{ }^{2}=.142\right]$, búsqueda de refuerzo alternativo $\left[F(1,113)=8498, p<0.005, \operatorname{eta}^{2}=.070\right]$.

Finalmente el tipo de universidad (laica-religiosa) tiene efecto sobre algunos factores relacionados con las estrategias de afrontamiento como apoyo religioso $[F(1,113)=6452$, $\mathrm{p}<0.05$, eta $\left.{ }^{2}=.054\right]$, autoinstrucciones $\left[F(1,113)=6873, \mathrm{p}<0.05\right.$, eta $\left.{ }^{2}=.057\right]$, ayuda para la acción $[F(1,113)=6105, p<0.05$, eta $=.051]$, reevaluación positiva y firmeza $[F(1,113)$ $=8297, p<0.005$, $\left.e t a^{2}=.068\right]$. A continuación podemos observar en la tabla 4 las medias para cada género y para cada Universidad (laica-religiosa). 
Tabla 4. Estadísticos descriptivos de factores de la escala de afrontamiento

\begin{tabular}{|c|c|c|c|c|c|c|c|}
\hline \multicolumn{4}{|c|}{ FACTORES } & \multicolumn{4}{|c|}{ FACTORES } \\
\hline \multicolumn{4}{|c|}{ F. Búsqueda de ayuda } & \multicolumn{4}{|c|}{ F. Acción dirigida a las causas } \\
\hline & Laica & Religiosa & Total & & Laica & Religiosa & Total \\
\hline Hombre & $2.67(.74)$ & $2.40(.74)$ & $2.64(.74)$ & Hombre & $2.42(.39)$ & $2.03(.24)$ & $2.38(.40)$ \\
\hline Mujer & $3.10(.57)$ & $3.28(.53)$ & $3.19(.55)$ & Mujer & $2.67(.46)$ & $2.77(.37)$ & $2.72(.42)$ \\
\hline Total & $2.85(.70)$ & $3.17(.62)$ & & Total & $2.53(.44)$ & $2.67(.43)$ & \\
\hline \multicolumn{4}{|c|}{ F. Apoyo religioso } & \multicolumn{4}{|c|}{ F. Autoinstrucciones } \\
\hline & Laica & Religiosa & Total & & Laica & Religiosa & Total \\
\hline Hombre & $1.86(.65)$ & $2.36(.95)$ & $1.91(.69)$ & Hombre & $3.05(.45)$ & $2.62(.57)$ & $3(.47)$ \\
\hline Mujer & $1.82(.63)$ & $2.24(.71)$ & $2.03(.70)$ & Mujer & $3.18(.36)$ & $3.01(.42)$ & $3.09(.40)$ \\
\hline Total & $1.84(.64)$ & $2.26(.73)$ & & Total & $3.10(.42)$ & $2.96(.46)$ & \\
\hline \multicolumn{4}{|c|}{ F. Ayuda para la acción } & \multicolumn{4}{|c|}{ F. Preparación para lo peor } \\
\hline & Laica & Religiosa & Total & & Laica & Religiosa & Total \\
\hline Hombre & $2.34(.51)$ & $1.76(.36)$ & $2.28(.52)$ & Hombre & $2.72(.43)$ & $2.33(.42)$ & $2.68(.44)$ \\
\hline Mujer & $2.45(.50)$ & $2.34(.56)$ & $2.40(.53)$ & Mujer & $2.78(.52)$ & $2.83(.42)$ & $2.80(.47)$ \\
\hline Total & $2.39(.50)$ & $2.27(.57)$ & & Total & $2.75(.47)$ & $2.76(.45)$ & \\
\hline \multicolumn{4}{|c|}{ F. Reevaluación positiva y firmeza } & \multicolumn{4}{|c|}{ F. Comunicación de sentimientos y apoyo social } \\
\hline & Laica & Religiosa & Total & & Laica & Religiosa & Total \\
\hline Hombre & $3.22(.51)$ & $2.67(.43)$ & $3.16(.52)$ & Hombre & $2.71(.75)$ & $2.24(.51)$ & $2.66(.74)$ \\
\hline Mujer & $3.16(.41)$ & $3.04(.35)$ & $3.10(.38)$ & Mujer & $3.19(.61)$ & $3.29(.61)$ & $3.24(.61)$ \\
\hline Total & $3.19(.46)$ & $2.99(.37)$ & & Total & $2.92(.73)$ & $3.15(.69)$ & \\
\hline \multicolumn{4}{|c|}{ F. Búsqueda de refuerzo alternativo } & & & & \\
\hline & Laica & Religiosa & Total & & & & \\
\hline Hombre & $2.84(.38)$ & $2.45(.77)$ & $2.80(.44)$ & & & & \\
\hline Mujer & $2.88(.42)$ & $3.04(.33)$ & $2.96(.38)$ & & & & \\
\hline Total & $2.86(.39)$ & $2.96(.44)$ & & & & & \\
\hline
\end{tabular}

\section{Discusión}

Los resultados nos han permitido cumplir con los objetivos y verificar las hipótesis de investigación planteadas. Como propusimos, hemos descrito el perfil de los alumnos en resiliencia y estrategias de afrontamiento. Para la muestra total del alumnado, se destaca que tiene una puntuación media-alta en resiliencia global (Miller, 1995; Rodríguez y Valdivieso, 2008) destacando en aspectos como la percepción de control donde creen que pueden ser ellos quienes controlen las situaciones y cambio relacionado con la posibilidad de establecer relaciones en las que apoyarse y de ser flexibles para adaptarse a situaciones nuevas. En cuanto al perfil de estrategias de afrontamiento la muestra total de alumnos destaca por utilizar estrategias centradas en el problema frente a aquellas más centradas en el aspecto emocional (locus externo) para solucionar los problemas. Estos aspectos son positivos por 
cuanto que favorecen la búsqueda de soluciones y la adopción de responsabilidad para solucionar los problemas favoreciendo el desarrollo de autonomía y conciencia ante la situación de estrés.

Aceptamos la segunda hipótesis para la que consideramos que las estrategias de afrontamiento centradas en el problema tendrían una relación positiva y significativa con la escala de resiliencia, ya que como señalan las investigaciones (De la Fuente y cols., 2013; Orozco, 2007), las estrategias de coping son parte del elenco de factores protectores resilientes.

Aceptamos parcialmente la tercera hipótesis ya que no se ha encontrado para esta muestra un efecto de las dos variables universidad $x$ sexo sobre la resiliencia de los alumnos pero sí sobre la dimensión estrategias de afrontamiento centradas en el problema donde comprobamos que para esta muestra, las chicas que estudian en la universidad de carácter religioso destacan en el uso de estas estrategias. También encontramos un efecto de ambas variables juntas (universidad $x$ sexo) sobre algunos factores relacionados con las estrategias de afrontamiento como acción dirigida a las causas y búsqueda de refuerzo alternativo donde nuevamente las chicas que estudian en la universidad de carácter religioso son quienes utilizan ambos aspectos ante situaciones de estrés.

En cuanto al efecto del género o sexo aceptamos parcialmente la hipótesis planteada. No encontramos efecto del sexo sobre la puntuación global de resiliencia (De la Fuente y cols., 2013; McLafferty, Mallet y McCauley, 2012) ni sobre sus cinco factores. Sólo observamos este efecto sobre la dimensión estrategias de afrontamiento centradas en el problema y sobre algunos factores de la escala de afrontamiento donde las chicas destacarían en los siguientes aspectos frente a los chicos: buscar ayuda para solucionar los problemas, expresar más sus sentimientos, analizar las causas y posibles consecuencias, aprender de situaciones pasadas, establecer planes de acción, los pasos, pedir ayuda a algún profesional, prepararse para lo peor, comunicar cómo te sientes y buscar refuerzo alternativo. En cuanto a los factores, se observa que los chicos son quienes utilizan más estrategias de reevaluación positiva y firmeza mientras que las chicas utilizan en mayor medida que los chicos todas las demás que se han señalado. 
Sobre el efecto del tipo de universidad, aceptamos la hipótesis planteada ya que se observan efectos en la resiliencia y en el tipo de estrategias de afrontamiento que se utilizan. Para la resiliencia, observamos el efecto en los factores tenacidad y espiritualidad donde señalamos que los alumnos de la universidad laica destacan en el primero mientras los alumnos de la universidad de carácter religioso lo hacen en el segundo como esperábamos. Relacionado con las estrategias de afrontamiento, observamos un efecto de la universidad sobre las estrategias centradas en el problema donde los alumnos de la universidad religiosa puntúan más alto en el uso de este tipo de estrategias. En cuanto a los factores, también señalamos que los alumnos de la universidad religiosa puntúan más alto en el apoyo religioso, (Ejemplo ítems: confían en que Dios remedie la situación problemática, oran cuando tienen problemas, tienen fe en que las cosas cambien), mientras que los alumnos de la universidad laica en ítems como analizar las causas del problema y posibles consecuencias, aprender de situaciones similares, buscar ayuda y consejo para solucionar los problemas y ver el lado positivo cuando se encuentran ante situaciones de estrés.

\section{Conclusiones}

En el marco de la creciente literatura que destaca el rol de los factores no cognitivos en el rendimiento académico y la persistencia (Leary y DeRosier, 2012), los estudios sobre la resiliencia en la universidad tienen un claro interés (Munro y Pooley, 2009). Este trabajo es una pequeña aportación a la escasez de estudios sobre resiliencia y afrontamiento en un contexto de estrés como es el universitario.

Los hallazgos de este estudio indican que la resiliencia y las estrategias de afrontamiento son constructos relacionados como cabía esperar y se confirma que la relación es mayor con las estrategias de afrontamiento activo lo que muestra un patrón adaptativo en el ámbito universitario (De la Fuente y cols. 2013; Li, in press; Terzi, 2013).

Este estudio piloto indica que se producen algunas variaciones en el perfil de resiliencia percibida y en las estrategias de afrontamiento en función del tipo de universidad en que se encuentran y en función del género, lo que confirmaría la especificidad de los constructos analizados en línea con lo que se señala en la literatura (Fergus y Zimmerman, 2005). Sería conveniente que se tuvieran en cuenta en el diseño de programas de intervención (McLafferty y cols., 2009), en particular en lo que respecta a los varones que muestran menor 
uso de estrategias de afrontamiento centrado en el problema. También resulta de interés la constatación de diferencias en los factores de espiritualidad de los dos constructos analizados en función del tipo de universidad (laica/religiosa) lo que podría ser estudiado con más profundidad en futuras investigaciones dada la creciente atención en la literatura por el factor espiritualidad como protector de la resiliencia (Davino, 2013; Foy, Drescher, Watson, 2011; Pargament, Cummings, 2010; Smith,Webber y DeFrain, 2013).

En resumen, los hallazgos sugieren que las escalas empleadas son consistentes y se pueden usar para mejorar nuestra comprensión de esta población y para ayudar a los alumnos a tomar conciencia e identificar sus fortalezas y desarrollar recursos de protección frente a situaciones de estrés. Por otra parte, tanto las escalas como los resultados obtenidos resultarán útiles para las universidades que deseen incorporar el examen de las variables no cognitivas en los procesos de admisión, como ya hacen algunas universidades como la universidad de Notre Dame (EEUU) que emplea medidas de resiliencia, entre otras (OnlineSchools.org, 2014). Además, tienen interés en cuanto que pueden proporcionar claves para orientar la labor educativa, preventiva y de intervención de las universidades de cara a ayudar a todos los estudiantes a conseguir una buena transición y ajuste en este ámbito tan decisivo para sus vidas futuras (Chung, 2011; Davino, 2013; De la Fuente y cols. in review; Hassim y cols., 2013; McCann y Hicks, 2011; Orozco, 2007; Prinyapol, 2003; Steinhardt y Dolbier, 2008; Terzi, 2013).

Por otra parte este trabajo no está exento de limitaciones. Entre las que cabe destacar: el pequeño tamaño de la muestra; los análisis estadísticos efectuados que, aunque de interés, no permiten establecer relaciones de causa efecto entre los constructos examinados y los problemas típicos de las escalas de autoinforme sujetas a la percepción subjetiva y, por tanto, a sus sesgos. Los resultados no son generalizables a la población universitaria, pero apuntan tendencias significativas que sería conveniente examinar con un aparato metodológico y de muestra mayor. 


\section{Agradecimientos}

Proyecto ref. EDU2011-24805 (2012-2014). Estrategias motivacionales-Afectivas de Autorregulación personal y de Afrontamiento del Estrés en el proceso de Enseñanza-Aprendizaje Universitario. MICINN (Spain) and FEDER Fund (UE).

\section{Referencias}

Artuch, R. (2014). Resiliencia y autorregulación de la conducta en jóvenes navarros en riesgo de exclusión social, que acuden a Programas de Cualificación Profesional Inicial. Tesis Doctoral Facultad de Educación y Psicología. Universidad de Navarra Pamplona http://dspace.unav.es/dspace/handle/10171/36188.

Benard, B. (1991). Fostering Resiliency in Kids: Protective Factors in the Family, School, and Community. San Francisco: Far West Laboratory for Educational.

Borghans, L., Duckworth, A. L., Heckman, J. J. y Ter Weel, B. (2008). The Economics and Psychology of Personality Traits, Journal of Human Resources, 43, 972-1059. DOI: 10.3368/jhr.43.4.972

Campbell-Sills, L., Cohan, S. L. y Stein, M. B. (2006). Relationship of resilience to personality, coping, and psychiatric symptoms in young adults. Behaviour Research and Therapy, 44(4), 585-599. DOI: 10.1016/j.brat.2005.05.001

Claxton, G., Chambers, M.; Powell, G. y Lucas, B. (2011). The Learning Powered SchoolPioneering 21st Century Education. Bristol: TLO Limited.

Conley, D.T. (2013). Rethinking the Notion of Noncognitive. Education Week. Retrieved from http://www.edweek.org/ew/articles/2013/01/23/18conley.h32.html. Extraído el 10 de septiembre de 2014.

Connor, D. y Davidson, R. (2003). The Connor-Davison Resilience Scale, CD-RISC. Depression and Anxiety, 18, 76-82.

Connor, K. M., Davidson, J. R. T. y Lee, L. C. (2003). Spirituality, resilience, and anger in survivors of violent trauma: A community survey. Trauma Stress, 16(5), 487-94. DOI: 10.1023/A:1025762512279

Costa, A.L. y Kallick, B. (2000). Habits of Mind. A development series. Alexandria, VA: ASCD.

Costa, A.L. y Kallick, B. (2014). Dispositions: Reframing Teaching and Learning. Thousand, Oaks, $C A$ : Corwin Press.

Chorot, P. y Sandín, B. (1993). Escalas de Estrategias de Coping revisado (EEC-R). [Scales of revised Coping strategies]. Madrid: UNED. 
Chung, H. (2011). Resiliency and character strengths among college students. ProQuest, UMI Dissertation Publishing, (Doctoral Dissertation, 2008).

Davino, D. (2013). Resilient First-Generation College Students: A Multiple Regression Analysis Examining the Impact of Optimism, Academic Self-Efficacy, Social Support, Religiousness, and Spirituality on Perceived Resilience (Dissertation $\mathrm{n}^{\circ}$ 3571591). Virginia: West Virginia University.

De la Fuente, J. (2014a). The e-Coping of Academic Stress. Almería: University of Almería.

De la Fuente, J. (2014b). Competencia para aprender, estudiar y rendir bajo estrés. Guía de auto-ayuda para universitarios, estudiantes y opositores. Almería: Education \& Psychology I+D+i.

De la Fuente, J., Cardelle-Elawar, M., Martínez-Vicente, J.M., Zapata, L. y Peralta, F.J. (2013). Gender as a determining factor in the coping strategies and resilience of university students. En R. Haumann and G. Zimmer (Eds), Handbook of Academic Performance (pp. 205-217). New York: Nova Science Publishers, Inc.

De la Fuente, J., Zapata, L., Putwain, D., González-Torres, M.C. y Artuch, R. (2013). Relationship between resilience and strategies for coping with stress at university. CIEAE 2013. Lisboa: Institute of Education, July, 15-18.

De la Fuente, J., Solinas, G., Fadda, S. y Zapata, L. (in review). The "E-COPING" stress management tool for university improving mental health through an online selfassessment and self-help utility in university students. En M. M. Cruz-Cunha y I.M. Miranda (eds.), Encyclopedia of Health and Telemedicine. USA: IGI Global.

De Ridder, D.T.D., Lensvelt-Mulders, G., Finkenauer, C., Stok, F.M. y Baumeister, R.F. (2012). Taking stock of self-control: A meta-analysis of how trait self-control relates to a wide range of behaviors. Personality and Social Psychology Review, 16, 76-99. DOI: $10.1177 / 1088868311418749$

Doll, B. Zucker, S. y Brehm, K. (2014). Resilient classrooms. Creating Healthy Environments for Learning. New York, NY: Guilford Press

Duckworth, A. L. y Carlson, S. M. (2013). Self-regulation and school success. En B. W. Sokol, F. M. E. Grouzet y U. Muller (Eds.), Self-regulation and autonomy: Social and developmental dimensions of human conduct (pp. 208-230). New York: Cambridge University Press.

Duckworth, A. L. y Seligman, M. E. P. (2005). Self-discipline outdoes IQ in predicting academic performance of adolescents. Psychological Science 16 (12), 939-944. DOI: 10.1111/j.1467-9280.2005.01641.x

Duckworth, A. L., Peterson, C., Matthews, M. D. y Kelly, D. R. (2007). Grit: Perseverance and passion for long-term goals. Journal of Personality and Social Psychology, 92(6), 1087-1101. DOI: 10.1037/0022-3514.92.6.1087

Durlak, J.A., Weissberg, R.P., Dymnicki, A.B., Taylor, R.D. y Schllinger, K.B. (2011) The impact of enhancing students' social and emotional learning: a meta-analysis of 
school-based universal interventions. Child Development, 82(1):405-32. DOI: 10.1111/j.1467-8624.2010.01564.x

Dweck, C. S. (2006). Mindset: The new psychology of success. New York: Random House.

Dweck, C. S., Walton, G. M. y Cohen, G. L. (2011). Academic tenacity. White paper prepared for the Gates Foundation. Seattle, WA.

Dziegielwski, S. F., Turnage, B. y Roest-Marti, S. (2004). Addressing stress with social work students: A controlled evaluation. Journal of Social Work Education, 40 (1), 105-119. DOI: $10.1080 / 10437797.2004 .10778482$

Egalité, A.J., Mills, J.N. y Greene, J.P (2014). The softer side of learning: measuring

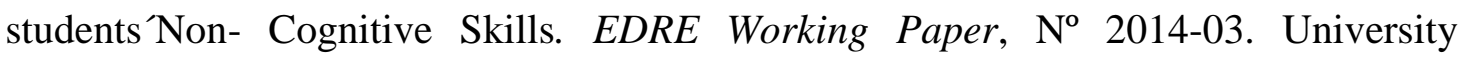
Arkansas: The Department of Education Reform.

Farrington, C.A., Roderick, M., Allensworth, E., Nagaoka, J., Keyes, T.S., Johnson, D.W. y Beechum, N.O. (2012). Teaching adolescents to become learners. The role of noncognitive factors in shaping school performance: A critical literature review. Chicago: University of Chicago Consortium on Chicago School Research.

Fergus S. y Zimmerman, M.A. (2005). Adolescent resilience: A framework for understanding healthy development in the face of risk. Annual Review Public Health, 26, 399-419. DOI: 10.1146/annurev.publhealth.26.021304.144357

Foy, D.W., Drescher, K.D. y Watson, P.J. (2011) Religious and spiritual factors in resilience. En St. M. Southwick , B. T. Litz , D.Ch., M.J. Friedman. Resilience and Mental Health. Challenges Across the Lifespan, (pp. 90-102). Cambridge: Cambridge University Press.

Glennie, E.J. (2010). Coping and resilience. En Rosen, J.A., Glennie, E.J., Dalton, B.W., Lennon, J.M., Bozick, R.N. (2010). Noncognitive Skills in the Classroom: New Perspectives on Educational Research (pp. 169-194) RTI Press Publication No. BK0004-1009. Research Triangle Park, NC: RTI Press.

Goldstein, S. y Brooks, R. B. (Eds.) (2013). Handbook of Resilience in Children. New York, NY: Springer, $2^{\circ}$ ed.

González -Torres, M.C. (2011). Hermanos resilientes: Taller para afrontar problemas de manera positiva. En O. Lizasoain, M.C. González-Torres; C. Iriarte Redín, F. Peralta, A. Sobrino; E. Chocarro de Luis (eds.), Hermanos de personas con discapacidad intelectual: Guía para el análisis y propuestas de apoyo. (pp.115-165). Logroño: Siníndice.

González-Torres, M.C. (2012). Más allá de la motivación: cultivar la voluntad de aprender para hacer frente a las demandas escolares, favorecer el éxito escolar y el desarrollo positivo de los estudiantes. IDEA (Revista del Consejo Escolar de Navarra) 39, 31-45.

Gutman, L. M. y Schoon, I. (2013). The impact of non-cognitive skills on outcomes for young people: literature review. Education Endowment Foundation (EEF) and the UK Cabinet Office. 
Hassim. T., Strydom, C. y Stridom, H. (2013). Resilience in a group of first-Year Psychosocial Science Students at the North-West University (Potchefstroom Campus). West East Journal of Social Sciences-April, 2.

Heckman, J. J. y Rubinstein, Y. (2001). The Importance of Noncognitive Skills: Lessons from the GED Testing Program. American Economic Review, 91 (2), 145-149. DOI: 10.1257/aer.91.2.145

Heckman, J. J., Stixrud, J. y Urzua, S. (2006). The effects of cognitive and noncognitive abilities on labor market outcomes and social behavior. Journal of Labor Economics, 24(3), 411-482. DOI: 10.3386/w12006

Johnson, E.L. (2011). Protective factors and levels of resilience among college student. Dissertation Alabama: University of Alabama.

Jordan, B. (2010). Educational resilience in primary school children in South Australia: an investigation. Thesis (Ph.D.). Australia: University of Adelaide.

Lazarus, R. S. y Folkman, S. (1984). Stress, appraisal and coping. New York, NY: Springer.

Leary, K.A. y DeRosier, M.E. (2012). Factors Promoting Positive Adaptation and Resilience during the Transition to College. Psychology, 3 (12A), 1215-1222. DOI: 10.4236/psych.2012.312A180

Li, M.H. (2008). Helping College Students Cope: Identifying Predictors of ActiveCoping in Different Stressful Situations. Journal of Psichiatry, Psychology and Mental health, 2(1), 1-15.

Li. M.H. (in press). Relationships among stress coping, secure attachment, and the trait of resilience among Taiwaness college students. College Student Journal, 41.

Luthar, S. S., Cicchetti, D. y Becker, B. (2000). The construct of resilience: A critical evaluation and guidelines for future work. Child Development, 71(3), 543-562. DOI: 10.1111/1467-8624.00164

Luthar, S. S., Sawyer, J. A. y Brown, P. J. (2006). Conceptual Issues in Studies of Resilience: Past, Present, and Future Research. Annals of the New York Academy of Science, 1094(1), 105-115. DOI: 10.1196/annals.1376.009

Martin, A.J. y Marsh, H.W. (2008). Academic buoyancy: Towards an understanding of students' everyday academic resilience. Journal of School Psychology, 46(1), 53-83. DOI: $10.1016 /$ j.jsp.2007.01.002

Masten, A. S. (2001). Ordinary magic: Resilience processes in development. American Psychologist, 56(3), 227-238. DOI: 10.1037//0003-066X.56.3.227

Masten, A. S. y Coatsworth, J. D. (1998). The development of competence in favorable and unfavorable environments: Lessons from research on successful children. American Psychologist, 53(2), 205-220. DOI: 10.1037/0003-066X.53.2.205

McCann, A. y Hicks, R. E. (2011). Resilience in university students: Academic success, recollected parental style, and coping strategies. En K.M. Gow y M.J. Celinski (Eds.), Wayfinding through life's challenges (pp. 485-499). New York, NY: Nova Science. 
McLafferty, M.; Mallet, J. y McCauley, V. (2012). Coping at university: the role of resilience, emotional intelligence, age and gender. Journal of Quantitative Psychological Research, 1, 1-6.

Miller, M. (1995). Sources of Resilience Outcomes. Paper presented at the International Convention of the Council for Exceptional Children. Indianapolis.

Munro, B. y Pooley, J.A. (2009). Differences in resilience and University adjustment between school leaver and mature Entry university studentes. The Australian Community Psychologist, 21(1), 50-61.

OnlineSchools.org. Beyong Standardized Test: How Non-Cognitive Skills Indicate College, Career Success. http://www.onlineschools.org/beyond-the-sat/ Extraído el 28 de Agosto de 2014.

Orozco, V. (2007). Ethnic identity, perceived social support, coping strategies, university environment, cultural congruity, and resilience of Latinalo college students. Dissertation. Ohio: The Ohio State University. http://proquest.umi.com/pqdweb?index $=6 \&$ did $=1390281351 \&$ SrchMode $=1 \&$ sid... 2/12/2008.

Pargament, K.T. y Cummings, J. (2010). Anchored by faith: Religion as a resilience factor. En J.W. Reich, A.J. Zautra y J.S. Hall (Eds.), Handbook of adult resilience, (pp. 193210). New York: Guilford Press.

Patterson, J.M. (2002). Understanding family resilience. Journal of Clinical Psychology, 58(3), 233-246. DOI: 10.1002/jclp.10019

Perkins, D.N. y Tishman, S. (2001). Dispositional aspects of intelligence. En S. Messick, y J.M. Collis (Eds.) Intelligence and Personality: Bridging the Gat in Theory and mesasurement. (pp. 233-257). Mahwah, New Jersey: Erlbaum.

Prince-Embury, S. y Saklofske, D.H. (2013). Resilience in Children, Adolescents, and Adults: Translating Research into Practice. New York, NY: Springer.

Prinyapol, P. (2003). Relationship between Resilience, Perception of Life Adversity and Coping Strategies of University Students. Master Thesis, Chiangmai University.

Ritchhart, R. (2001). From IQ to IC: A dispositional View of Intelligence. Roeper Review, 23 (3), 143-150. DOI: 10.1080/02783190109554086

Rodríguez, C.R. y Valdivieso, A.G. (2008). El éxito escolar en alumnos en condiciones adversas. Revista latinoamericana, 38 (81-2), 81-106.

Ross, S.E., Niebling, B.C. y Heckert, T.M. (1999). Sources of stress among college students. College Student Journal, 33, 312-317.

Sagone, E. y De Caroli. M.E. (2014). A Correlational Study on Dispositional Resilience, Psychological Well-being, and Coping Strategies in University Students. American Journal of Educational Research 2(7), 463-471. DOI: 10.12691/education-2-7-5

Smith, L., Webber, R. y DeFrain, J. (2013). Spiritual Well-Being and Its Relationship to Resilience in Young People. A Mixed Methods Case Study Sage Open, 3 (2), 1-16. DOI: $10.1177 / 2158244013485582$ 
Smith-Osborne, A. (2007). Life Span and Resiliency Theory: A Critical Review. Advances in social work, $8(1), 152-168$.

Steinhart , M.y Dolbier, C. (2008). Evaluation of a resilience intervention to enhance coping strategies and protective factors and decrease symptomatology. Journal of American College Health, 56(4), 445-53. DOI: 10.3200/JACH.56.44.445-454

Suto, I. (2013). 21stCentury skills: Ancient, ubiquitous, enigmatic? Research Matters: A Cambridge Assessment Publication. Cambridge: University of Cambridge.

Terzi, S. (2013). Secure attachment style, coping with stress and resilience among university students. The Journal of Happiness \& Well-Being, 1(2), 97-109.

Tough, P. (2012). How Children Succeed: Grit, Curiosity, and The Hidden Power of Character. New York: Houghton Mifflin Harcourt.

Vargas-Villalobos, L. y González-Torres, M.C. (2009). La revitalización de la Educación del Carácter en el ámbito psicoeducativo actual: Aportaciones desde las Ciencias de la Prevención y la Psicología Positiva. Electronic Journal of Research in Educational Psychology, 7(3), 1379-1418

Waxman, H.C., Gray, J.P. y Padron, Y.N. (2003). Review of Research on Educational Resilience: (Research Reports No. 11). Santa Cruz: Center for Research on Education, Diversity, \& Excellence University of California.

Werner, E. E. (2007). Resilience and protective factors in the lives of individualswho were children and youths in World War II. En I. Fooken y J. Zinnecker (Eds.), Trauma und Resilienz (pp. 47-55). Munich: Juventa Verlag.

Werner, E. E. y Smith, R. S. (1982). Vulnerable but not invincible: A longitudinal study of resilient children and youth. New York, NY: Donnelley and Sons, Inc.

West, M. R., Gabrieli, C. F. O., Finn, A. S., Kraft, M. A. y Gabrieli, J. D. E. (2014).What Effective Schools Do: Stretching the Cognitive Limits on Achievement, Education Next, 14(4), 72-79.

West, M., Kraft, M.A., Finn, A.S., Martin, R., Duckworth, A.L., Gabrieli, C.F.O. y Gabrieli, J.D.E. (2014). Promise and paradox: Measuring students's non-cognitive skills and the impact of schooling. Working Paper. Harvard, MA: Harvard University.

Windle, G., Bennett, K.M. y Noyes, J. (2011). A methodological review of resilience measurement scales. Health and Quality of Life Outcomes, 9(8), 1-18. DOI:10.1186/1477-7525-9-8

Wolters, C. (2003). Regulation of motivation: Evaluating an underemphasized aspect of selfregulated learning. Educational Psychologist, 38, 189-205. DOI: 10.1207/S15326985EP3804_1

Yates, T.M. y Masten, A.S. (2004). Fostering the future: Resilience theory and the practice of positive psychology. En P.A. Linley y S. Joseph (Eds.), Positive Psychology in practice (pp. 521-539). Hoboken, NJ: Wiley. 
Yeager, D. S. y Dweck, C.S. (2012). Mindsets That Promote Resilience: When Students Believe that Personal Characteristics Can Be Developed. Educational Psychologist, 47 (4) 302-314. DOI: 10.1080/00461520.2012.722805

Zapata, L. (2013). Self-regulation, learning and coping in stressful contexts of higher education. Doctoral Dissertation. Almería: Facultad de Humanidades y Psicología. Universidad de Almería.

Zolkoski, S. M. y Bullock, L.M. (2012). Resilience in children and youth: A review. Children and Youth Services Review, 34, 2295-2303. DOI: 10.1016/j.childyouth.2012.08.009 